\title{
Soil Medium Electromagnetic Scattering Model for the Study of Wireless Underground Sensor Networks
}

\author{
Frank Kataka Banaseka ${ }^{D},{ }^{1,2}$ Hervé Franklin, ${ }^{2}$ Ferdinand A. Katsriku, ${ }^{2}$ \\ Jamal-Deen Abdulai, ${ }^{2}$ Akon Ekpezu, ${ }^{2}$ and Isaac Wiafe ${ }^{2}$ \\ ${ }^{1}$ University of Professional Studies, Accra, Department of Information Technology, Ghana \\ ${ }^{2}$ University of Ghana, Legon, Department of Computer Science, Ghana \\ Correspondence should be addressed to Frank Kataka Banaseka; frank.banaseka@upsamail.edu.gh
}

Received 9 September 2020; Revised 26 November 2020; Accepted 22 December 2020; Published 4 January 2021

Academic Editor: Pei-Wei Tsai

Copyright (C) 2021 Frank Kataka Banaseka et al. This is an open access article distributed under the Creative Commons Attribution License, which permits unrestricted use, distribution, and reproduction in any medium, provided the original work is properly cited.

\begin{abstract}
In recent years, there has been keen interest in the area of Internet of Things connected underground, and with this is the need to fully understand and characterize their operating environment. In this paper, a model, based on the Peplinski principle, for the propagation of waves in soils that takes into account losses attributable to the presence of local inhomogeneity is proposed. In the work, it is assumed that the inhomogeneities are obstacles such as stones or pebbles, of moderate size, all identical and randomly distributed in space. A new wave number is obtained through a combination of the multiple scattering theory and the Peplinski principle. Since the latter principle considers the propagation in a homogeneous medium (without obstacles), the wave number it provides is inserted into the one resulting from the former, the multiple scattering theory. The effective wave number thus obtained is compared numerically with that of Peplinski alone on the one hand and with that of multiple scattering alone on the other hand. The phase velocity and the loss tangent are analyzed against the particle concentration at the low-frequency Rayleigh limit condition $(k a \lesssim 0.1)$ and against the frequency at two particle concentrations $(\boldsymbol{c}=\mathbf{0 . 2}$ and $\boldsymbol{c}=\mathbf{0 . 4})$, two particle radii $(\boldsymbol{a}=\mathbf{0 . 5 5} \mathrm{cm}$ and $\boldsymbol{a}=\mathbf{1 . 1 0} \mathrm{cm})$, and 5\% and 50\% volumetric water content of the soil. Path losses are also compared to each other to examine the effects on transmission of soil containing obstacles. The results obtained suggest that the proposed model has better accuracy in estimating the wave number than previously used schemes.
\end{abstract}

\section{Introduction}

Wireless underground sensor networks (WUSNs) are an emerging area that has gained the attention of many researchers. This is because WUSNs open up new possibilities for underground monitoring and communication; also, they will find application in agriculture, which is key to the developmental agenda of many emerging nations. For instance, a WUSN path loss model based on an accurate prediction of the complex dielectric constant (CDC) for precision agriculture is proposed and called WUSN-PLM [1]. These WUSNs are now being interconnected to form the "Internet of Underground Things (IoUT)" to depict the internet of devices connected underground. IoUT encompasses devices buried in soil or placed in open bounded spaces and are interconnected to facilitate sending of infor- mation out of agricultural fields and other underground environments to decision-making and control centres. In the same context, an analytical survey was performed on the current and potential application of the Internet of Things in arable farming, state-of-the-art technologies deployed, challenges of mobile devices in spatial data collection, highly varying environments, and task diversity, compared to other agricultural systems $[2,3]$.

The physical phenomenon underpinning the operation of IoUT is the propagation of electromagnetic waves in a soil medium. The characteristics of the operating environment will have a great impact on the performance of the network. Obstacles such as stones cause waves to be refracted or scattered in an underground environment. In addition, an increase in communication range and volumetric water content of soil due to irrigation or rain will lead to high signal 
path loss. Additionally, signal propagation characteristics in soil are dependent on the soil type and properties. Typically, a two-stage model is proposed based on the field characteristics of the antenna and considers four sources of path loss. The two-stage model has a different coefficient, which depends on the soil types in the near-field and far-field regions $[4,5]$.

To effectively characterize the propagation environment, accurate and robust models are required. Different models have been proposed in the literature for the study of electromagnetic wave propagation in various environments [6-14]. In particular, for the characterization of electromagnetic wave (EM) propagation in soil, a number of different models have been proposed in the literature $[6,11,12,14]$; however, models that take into consideration factors such as multipath propagation, volumetric water content of soil, and burial depth are dominant $[6,11]$. Furthermore, some of these models analyze the bit error rate for communication performance based on some modulation schemes and soil properties.

As an alternative to EM wave propagation in soil, Sun and Akyildiz [11] have proposed the magnetic induction technique. The magnetic induction technique is a promising communication technique for analyzing propagation in soil $[10,11]$. EM waves and the Friis equations [12] were used to analyze the channel model taking into consideration the direct, reflected, and lateral waves, multipath, soil composition, and water content. It was shown that the direct, reflected, and lateral waves are major contributors to signal attenuation in the soil environment [11]. In [14], a segmentation approach is used to sense soil moisture where the radio field is used as a sensor. Based on the Peplinski principle [15], the path loss for different volumetric water content levels at three different frequencies was calculated. In comparison with some related research proposed recently as shown in Table 1, this work seeks to analyze the transmission of electromagnetic waves in a soil medium taking into account the presence of obstacles that cause multiple scattering. A new wave number model is proposed with the combination of the Peplinski principle and multiple scattering in the soil medium. The new wave number is used in the computation of the path loss.

To the best of our knowledge, path loss expressions that consider explicitly scattering and in particular multiple scattering are yet to be reported. In this paper, based on the results presented in $[9,14]$ where a relation for the path loss is derived, we consider the problem of EM wave propagation [16-18] in a dense medium with scattering properties. In the present work, a model of the effective wave number is presented that accounts for absorption due to permittivity and multiple scattering occurring in soil because of the presence of buried obstacles such as stones, rocks, or pebbles. To achieve this, it is assumed that the medium which typically is polydispersed contains identical objects of similar size. This assumption allows the change in path loss to be readily estimated. The propagation constants derived from the effective wave number obtained are used for the calculation of the path loss. The results are compared with those previously reported in the literature.
In addition, a parametric study is also performed that shows the effects of the concentration of obstacles or the volumetric water content on signal attenuation. The phase velocity and the loss tangent are analyzed against the particle concentration at the low-frequency Rayleigh limit condition and against the frequency at two particle concentrations: 0.2 and 0.4 , two particle radii: $0.55 \mathrm{~cm}$ and $1.10 \mathrm{~cm}$, and $5 \%$ and $50 \%$ volumetric water content of the soil. The analysis is performed considering only the Peplinski principle on the one hand and the Peplinski principle with multiple scattering on the other. Results obtained indicate that the approach proposed in this study could provide significantly better results than previously obtained and lead to a better characterization of WUSN.

\section{Comparison with Some Related Research Proposed Recently}

In [14], Liedmann et al. presented the path loss of an average topsoil for different distances and typical IoT frequencies at two different VWCs, 5\% and 50\%, respectively. The higher the operating frequency, the higher the influence of rising VWC. Meanwhile, in this work, path loss is modeled based on absorption due to permittivity and multiple scattering from obstacles in soil. It is then analyzed against distance at the same frequencies of $433 \mathrm{MHz}$ and $868 \mathrm{MHz}$ and at the same VWC proportions. Path loss analysis in both works shows almost the same trends.

In [1], four sources of path losses are analyzed based on a proposed two-stage model with field characteristics of the antenna. The two-stage model has a different coefficient, which depends on the soil types in the near-field and farfield regions. Path losses against transmission distance are compared on dry soil for sandy clay\# 1 and for sandy clay\#2. Path losses against sensor burial depth for underground-toabove the ground and above the ground-to-underground channel models are also compared for $5 \mathrm{~m}, 10 \mathrm{~m}, 15 \mathrm{~m}$, and $20 \mathrm{~m}$ transmission ranges. Results in this work show the same trend of path loss.

At the same operating frequency of $433 \mathrm{MHz}$ in [4], the comparison is made between the measured path loss and that of Friis, Fresnel, and the proposed propagation models tested within clayey silt, dry sand, and wet sand media. The results showed the same trend of increasing growth of path loss as the transmission distance increases to $0.5 \mathrm{~m}, 1 \mathrm{~m}$, and beyond. Meanwhile, in this work, a comparison has been established for a revised path loss based on only the Peplinski principle and on Peplinski combined with multiple scattering for two operating frequencies $868 \mathrm{MHz}$ and $433 \mathrm{MHz}$. The transmission distance considered in our study is up to $5 \mathrm{~m}$.

\section{System Architecture and IoUT Application in Agriculture}

In agriculture, IoUT is envisaged to provide total field autonomy and enable more efficient food production solutions through not only in situ monitoring and self-reporting capabilities (soil moisture, salinity, temperature, etc.) but also the interconnection of existing field machinery like irrigation 
TABLE 1: Comparison of some related research proposed recently with our work.

\begin{tabular}{|c|c|c|}
\hline Reference & Related research proposed recently & Our work \\
\hline$[1]$ & $\begin{array}{l}\text { A WUSN path loss model for precision agriculture called } \\
\text { WUSN-PLM is proposed. The proposed model is based on an } \\
\text { accurate prediction of the complex dielectric constant (CDC). }\end{array}$ & $\begin{array}{l}\text { A new wave number model is proposed using the combination } \\
\text { of the Peplinski principle and multiple scattering in a soil } \\
\text { medium. The new wave number is used in the computation of } \\
\text { the path loss. }\end{array}$ \\
\hline$[2]$ & $\begin{array}{l}\text { Underground environment-aware MIMO is developed using } \\
\text { transmit and receive beamforming. }\end{array}$ & $\begin{array}{c}\text { Signal transmission with single input single output (SISO) } \\
\text { between a transmitter and a receiver. }\end{array}$ \\
\hline$[3]$ & $\begin{array}{l}\text { Analytical survey of the current and potential application of the } \\
\text { Internet of Things in arable farming, state-of-the-art } \\
\text { technologies deployed, challenges of mobile devices in spatial } \\
\text { data collection, highly varying environments, and task } \\
\text { diversity, compared to other agricultural systems. }\end{array}$ & $\begin{array}{l}\text { Internet of Underground Things (IoUT) application in } \\
\text { precision agriculture, envisaged to provide total field autonomy } \\
\text { and enable more efficient food production solutions through } \\
\text { not only in situ monitoring and self-reporting capabilities but } \\
\text { also the interconnection of existing field machinery like } \\
\text { irrigation systems, harvesters, and seeders. }\end{array}$ \\
\hline$[4]$ & $\begin{array}{l}\text { A two-stage model is proposed based on the field characteristics } \\
\text { of an antenna and considers four sources of path loss. The two- } \\
\text { stage model has a different coefficient, which depends on the } \\
\text { soil types in the near-field and far-field regions. }\end{array}$ & $\begin{array}{c}\text { Path loss is modeled based on absorption and multiple } \\
\text { scattering from obstacles in soil. The path loss is then analyzed } \\
\text { against distance at two typical IoT frequencies of } 433 \mathrm{MHz} \text { and } \\
868 \mathrm{MHz} \text {. We also showed the effect of VWC on the path loss } \\
\text { for two proportions, } 5 \% \text { and } 50 \% \text {. }\end{array}$ \\
\hline
\end{tabular}

systems, harvesters, and seeders [19] as shown in Figure 1. Real-time decision-making takes place in IoUT at a monitoring centre in the cloud, which receives information required from sensors, underground, and other field devices. It is worth noting that in this architecture communication may take place through the soil medium between the underground devices or may take place through air and plant foliage devices attached to the plant body. IoUT applications have challenging requirements that include impairments attributable to wireless communication through soil [2023 ] and machinery operating in remote crop fields and exposure to the elements. Progress in IoUT research will significantly impact and benefit many application areas such as plant monitoring and control, border patrol, underground mines and tunnels, and pipeline assessment $[6,20,22]$. A model of IoUT architecture is illustrated in Figure 1. Based on the required functionalities, the architecture may include components such as underground objects, base stations, mobile sinks, and cloud services. Below, we explain the properties or characteristics of the various components.

Underground objects (UOs) are made up of objects which are wholly or partially embedded within the subterranean environment and are equipped with wireless communication and sensing modules. They are constructed to be rugged enough to protect them against farming equipment, wild rodents, and extreme weather conditions. Their onboard sensors usually include a wide range of soil-related or weather-related sensing devices that can monitor phenomena such as soil temperature, chemical properties, and moisture. The communication schemes employed include Bluetooth, ZigBee, NFC, Wi-Fi, Sigfox, LoRa, LoRaWAN, Satellite, and cellular [24].

Base stations (BSs) have high processing power and communication facilities installed in permanent structures like weather stations or buildings and are used as gateways to transfer the data collected from wireless nodes to the cloud (the monitoring centre). While it is not essential to have a base station in a wireless communications framework, they can be key to the operation of a wireless network and in extending its communication range.

In wireless sensor network applications, data transfer can account for up to $70 \%$ of the energy cost. This has led to the use of mobile sinks for data collection. Mobile sinks are vehicles (tractors, irrigation systems, harvesters, seeders, and unmanned aerial vehicle drones) that move periodically or as required, around the defined fields where the sensors are deployed to collect data from the sensor nodes.

Cloud services are used for real-time processing of the field data as well as permanent storage of the collected data. The cloud service, as the decision-making centre of the system, helps in integrating collected data with existing databases. This also serves as the platform through which services and information are made available to users and stakeholders.

Within the IoUT architecture, a number of different communication links may be identified. This includes (a) underground-to-underground link in which the entire communication is confined underground, such a scheme might be used when the sensors need to exchange information or when a sensor needs to route data through a neighbouring sensor; (b) underground to above ground: this part of the communication is underground and the other part is above ground with the transmitter being buried underground and the receiver is above. This scheme is mainly used to send data collected by the sensors to control centres; (c) above ground to underground: here, the transmitter is above ground, and the receiver may be the sensor device underground. The scheme is mainly used for sending control signals; (d) above-ground surface communication. In this deployment, the sensors (transmitter and receiver) are partially buried in the ground as such the communication link is above ground but within the immediate vicinity of the soil, the wave will therefore propagate just above the surface of the ground. Arguably, existing over-the-air (OTA) wireless communication protocols face significant challenges in underground environments because they were originally not designed for 


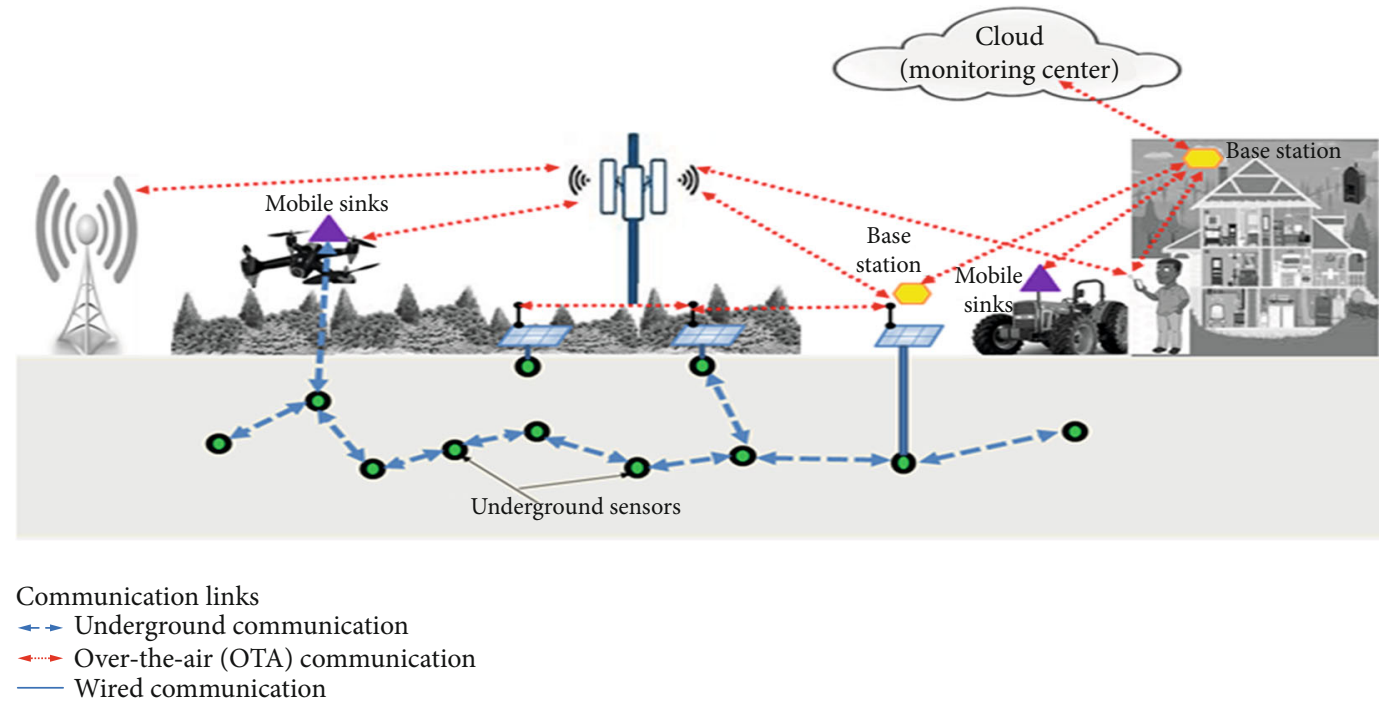

FIgURE 1: Architecture of IoUT.

these conditions. The electromagnetic wave attenuation in soil is much higher than that in air. This has a limiting effect on link quality. In above-ground-to-underground communication, it is critical to give due consideration to the important effects of reflection and refraction due to the ground surface.

\section{Absorption due to Permittivity}

In the following, a uniform random distribution of dielectric spheres with radii $a$ (also referred to as particles) of relative permittivity $\varepsilon_{p}$ embedded in a background medium (soil) of permittivity $\varepsilon$ is considered. Let $n_{o}$ be the number of spheres per unit volume and $\alpha$ the polarizability of each sphere. The polarization $\mathbf{P}$ (dipole moment per unit volume) is given by

$$
\mathbf{P}=\frac{n_{o} \alpha}{1-n_{o} \alpha / 3 \varepsilon} \mathbf{E},
$$

where $\mathbf{E}$ is the electric field inside the medium. Substituting (1) into the electric flux density $\mathbf{D}=\varepsilon \mathbf{E}+\mathbf{P}$ yields $\mathbf{D}=\varepsilon_{\mathrm{eff}} \mathbf{E}$, where

$$
\varepsilon_{e f f}=\varepsilon\left[\frac{1+2 n_{o} \alpha / 3 \varepsilon}{1-n_{o} \alpha / 3 \varepsilon}\right]
$$

are Clausius-Mossotti's formula of the effective permittivity. The formula for the polarizability is [3]

$$
\alpha=3 \varepsilon v_{o} \frac{\varepsilon_{p}-1}{\varepsilon_{p}+2},
$$

where $v_{0}=4 \pi a^{3} / 3$ is the volume of the sphere. Substituting $\alpha$ from (3), the relation for the effective permittivity yields Maxwell-Garnett's mixing formula:

$$
\varepsilon_{e f f}=\varepsilon\left(\frac{1+2 c y}{1-c y}\right),
$$

where $c=n_{o} v_{o}$ is the fractional volume occupied by the particles and $y$ is given by

$$
y=\frac{\varepsilon_{p}-1}{\varepsilon_{p}+2} .
$$

The effective wave number $K$ of the composite medium is given by $K=\omega \sqrt{\mu \varepsilon_{e f f}}$ or

$$
K^{2}=k^{2} \frac{1+2 c y}{1-c y}=k^{2}\left(1+\frac{3 c y}{1-c y}\right),
$$

where $k=\omega \sqrt{\mu \varepsilon}$ is the wave number for the background medium free of spheres and having a permittivity. The existence of an imaginary part for $K$ reflects the presence of the absorption phenomenon in the medium during the propagation. In (6), the permittivity can be derived from the Peplinski principle which is discussed later.

\section{Wave Attenuation due to Multiple Scattering in Soil: Quasicrystalline Approximation (QCA) in Dense Media}

Multiple scattering phenomena occur in soil in the presence of inhomogeneity or spheres which are randomly (or not) distributed. The presence of such inhomogeneities can have a significant effect on the propagation of the electromagnetic wave within the soil medium. It can be observed that (6) does not take into consideration the multiple scattering of the waves by the spheres. It seems appropriate to add a term taking into account this multiple scattering.

To address the difficult problem of wave propagation in soils and in particular the problem of multiple scattering, it is helpful to simplify the problem in the first instance. To do this, the scattering simulations can be performed on a test volume containing a large number of spheres but forming at the same time a small part (the representative elementary 
volume) of the whole system. The soils are normally polydisperse media since they contain scatterers of various shapes, sizes, and materials. In this work, for simplicity, it is assumed that all the scatterers are identical (monodispersity), the reason being that, by using the simplest equations of multiple scattering, we would be able to estimate the change in path loss. Figure 2 shows a plane electromagnetic wave incident onto a half-space of identical dielectric spheres. Such an incident wave will be subject to multiple scattering in the soil medium. To solve this problem of multiple scattering, the quasicrystalline approximation (QCA) and the $T$-matrix formalism were used by Tsang et al. [17].

In QCA, statistical configurational averaging using conditional averaging on positions is performed. The details of the calculation techniques leading to the formula of the effective wave number are out of scope for this study. Rather, our interest is on the formulas of the wave number that accounts for attenuation due to scattering.

Let $T_{n}$ denote the scattering coefficient for a sphere in mode $n$. Then, at the low-frequency Rayleigh limit $(k a \lesssim 0.1$ ), most of the contribution is attributable to $T_{1}(k a)$ (the mode $n=1$ corresponds to the electric dipole). In the context of the QCA, it follows that the effective wave number [16] is given by

$$
K^{2}=k^{2}\left\{1+\frac{3 c y}{1-c y}\left[1+i 2 c k^{3} a^{3} \frac{y}{1-c y}\left(1+4 \pi n_{0} J\right)\right]\right\}
$$

where

$$
J=\int_{0}^{\infty} r^{2}[g(r)-1] d r
$$

In (8), $g$ is the pair distribution function of one sphere position given the position of the other. This function can assume different functional forms, one of the most useful being the Percus-Yevick pair distribution function for hard spheres [19]. The pair distribution function depends on the scatterer size $a$ and the fractional volume $c$ of scatterers within the volume. Different forms of the Percus-Yevick pair distribution function have been discussed in [25] for various values of the fractional volume. In this study, the case of $c$ $=0.2$ and $c=0.4$ is considered. The Percus-Yevick function oscillates in the range of $r / 2 a$ with the degree of oscillation ranging from 1 to 3 , depending on the value of $c$, the fractional volume. The Percus-Yevick approximation for shortranged hard-sphere pair distribution function is considered [17]:

$$
n_{0} \int_{0}^{\infty} r^{2}[g(r)-1] d r=\frac{(1-c)^{4}}{(1+2 c)^{2}}-1
$$

A simplified formula of the effective wave number may be obtained as follows [18]:

$$
K^{2}=k^{2}\left\{1+\frac{3 c y}{1-c y}\left[1+i \frac{2 k^{3} a^{3}}{3} \frac{(1-c)^{4}}{(1+2 c)^{2}} \frac{y}{1-c y}\right]\right\}
$$

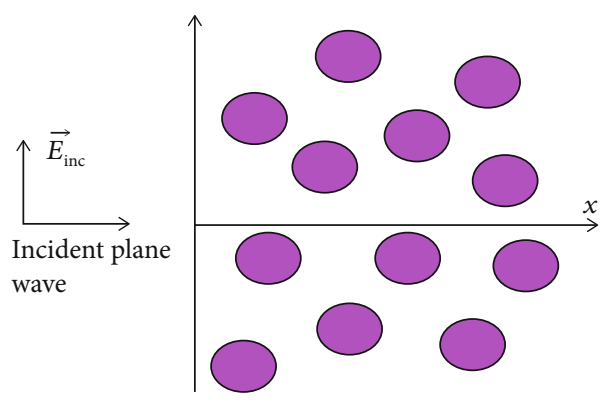

FIgURe 2: Plane electromagnetic wave normally incident on a halfspace of spherical dielectric scatterers of radius $a$.

(for values of $c$ ranging from 0.2 to 0.4 ). For $k a \leqq 0.1$ and at the operating frequency of $433 \mathrm{MHz}$ (which is within the $0.3-1.3 \mathrm{GHz}$ range of validity of Peplinski's dielectric mixing formula [(13)]), (10) is valid for soils with a random distribution of spheres having a radius not exceeding $1.1 \mathrm{~cm}$ as shown in Table 2. If the frequency is higher, a smaller radius is needed to satisfy the limiting condition $k a \leqslant 0.1$, which in essence, is an approximate condition [26].

Note that lower frequencies are required for adequate communication in the soil medium. However, reducing the operating frequency below $300 \mathrm{MHz}$ will increase the antenna size, which introduces practical challenges during WUSN implementation. Most wireless underground sensor boards such as MICA2 are designed to operate within 300 to $400 \mathrm{MHz}$ range. Ideally, operating frequencies of 300 and $900 \mathrm{MHz}$ are appropriate for preserving small antenna sizes [6]. This ensures that the sensors remain discrete, a property which is particularly useful for security applications. For a sparse medium (very small volume fraction), the PercusYevick function tends to the Hole-Correction formula [27], and the QCA in (10) is reduced to the EFA (Effective Field Approximation) represented by the simpler but less accurate formula:

$$
K^{2}=k^{2}\left\{1+3 c y\left[1+\frac{2}{3} i k^{3} a^{3} y\right]\right\} .
$$

Figure 3 shows the normalized phase velocity $\operatorname{Re}(k / K)$ and the loss tangent $2 \operatorname{Im} K / \operatorname{Re} K$ versus the concentration obtained from (10) for three values of $k a$. The permittivity of the background medium is $\varepsilon=8.854 \times 10^{-12}\left(\mathrm{~F} \mathrm{~m}^{-1}\right)$, and the relative permittivity of the spheres is $\varepsilon_{p}=3.2$. When the concentration increases, the phase velocity decreases monotonically while the loss tangent increases initially until a maximum value is attained when it begins to decrease.

In Figure 4, the permittivity of the background medium is assumed to be $\varepsilon=8.854 \times 10^{-12}\left(\mathrm{~F} \mathrm{~m}^{-1}\right)$, for spheres of radius $a \approx 0.55 \mathrm{~cm}$ and fractional volume concentrations of $c=0.2$ and $c=0.4$. The figure shows the normalized phase velocity $\operatorname{Re}(k / K)$ and the loss tangent $2 \operatorname{Im} K / \operatorname{Re} K$ given by $(10)$ versus the frequency. From Figure 4, it appears that regardless of the fractional volume concentration, $c=0.2$ or $c=0.4$, the normalized phase velocities remain constant. The waves propagate faster in the medium with the lowest 
TABLE 2: Evaluation of the maximum radius for the validity of (19).

\begin{tabular}{ccc}
\hline Frequency & $\begin{array}{c}\text { Condition } k a \\
\leqslant 0.1\end{array}$ & $\begin{array}{c}\text { Number of spheres per unit volume } \\
n_{0}=c / v_{0}\end{array}$ \\
\hline $433 \mathrm{MHz}$ & $a \lesssim 1.1 \mathrm{~cm}$ & $\approx 35,873($ if $c=0.2)$ \\
$868 \mathrm{MHz}$ & $a \leqslant 0.55 \mathrm{~cm}$ & $\approx 286,860$ (if $c=0.2)$ \\
\hline
\end{tabular}

concentration of spheres (radius $a \approx 0.55 \mathrm{~cm}$ ) but have the highest loss tangent.

Figure 5 depicts a plot of the normalized phase velocity and loss tangent against frequency. The permittivity of the background medium remains the same in Figure 4. The radius considered for the spheres is $a \approx 1.10 \mathrm{~cm}$ at concentrations $c=0.2$ and $c=0.4$. We can observe that the normalized velocities are practically identical and therefore do not depend on the radius. Secondly, the loss tangents take greater values in the reduced range of $300 \mathrm{MHz}-500 \mathrm{MHz}$. Table 3 provides a summary of the values used in the calculation of the wave number by QCA.

Peplinski et al. [15] reported the development of a semiempirical dielectric model for soils, covering the $0.3-1.3 \mathrm{GHz}$ range. The model provides expressions for the real and imaginary parts of the relative dielectric constant of a soil medium in terms of the soil's textural composition (sand, silt, and clay fractions), the bulk density and volumetric moisture content of the soil, and the dielectric constant of water, the specified microwave frequency, and physical temperature. A comparison of experimental results measured in this study with predictions based on the semiempirical model shows that the model developed underestimates the real part of the dielectric constant for cases where the moisture content of the soil is high.

Assuming a complex-valued permittivity $\varepsilon=\varepsilon^{\prime}-i \varepsilon^{\prime \prime}$, the propagation constants (the attenuation constant $\varsigma$ and the phase shift constant $\omega$ ) are given by $[2,22]$

$$
\gamma=\varsigma+i \omega
$$

where

$$
\begin{gathered}
\varsigma=\operatorname{Re}(\gamma)=\omega \sqrt{\frac{\mu \varepsilon^{\prime}}{2}\left[\sqrt{1+\left(\frac{\varepsilon^{\prime \prime}}{\varepsilon^{\prime}}\right)^{2}}-1\right]}, \\
\Phi=\operatorname{Im}(\gamma)=\omega \sqrt{\frac{\mu \varepsilon^{\prime}}{2}\left[\sqrt{1+\left(\frac{\varepsilon^{\prime \prime}}{\varepsilon^{\prime}}\right)^{2}}+1\right]} .
\end{gathered}
$$

According to the Peplinski principle, the relative dielectric properties of soil in the 0.3 to $1.3 \mathrm{GHz}$ band can be estimated as follows:

$$
\varepsilon^{*}=\varepsilon^{\prime}-i \varepsilon^{\prime \prime},
$$

where

$$
\begin{gathered}
\varepsilon^{\prime}=1.15\left[1+\frac{\rho_{b}}{\rho_{s}}\left(\varepsilon_{s}^{\alpha^{\alpha}}-1\right)+m_{v}^{\beta} \varepsilon_{f_{w}^{\prime \alpha}}^{\prime \alpha}-m_{v}\right]^{1 / \alpha^{\prime}}-0.68, \\
\varepsilon^{\prime \prime}=\left[m_{v}^{\beta^{\prime \prime}} \varepsilon_{f_{w}^{\prime \prime}}^{\alpha^{\prime}}\right]^{1 / \alpha^{\prime}} .
\end{gathered}
$$

In the above,

$$
\begin{gathered}
\varepsilon_{f w}^{\prime}=\varepsilon_{w \infty}+\frac{\varepsilon_{w 0}-\varepsilon_{w \infty}}{1+\left(2 \pi f \tau_{w}\right)^{2}}, \\
\varepsilon_{f w}^{\prime \prime}=\frac{2 \pi f \tau_{w}\left(\varepsilon_{w 0}-\varepsilon_{w \infty}\right)}{1+\left(2 \pi f \tau_{w}\right)^{2}}+\frac{\sigma_{\text {eff }}}{2 \pi \varepsilon_{0} f} \frac{\left(\rho_{s}-\rho_{b}\right)}{\rho_{s} m_{v}}
\end{gathered}
$$

represent the real and imaginary parts of the relative dielectric constant of water, with $\varepsilon_{w \infty}$ the high-frequency limit of $\varepsilon_{f w}^{\prime}, \varepsilon_{w 0}$ the static dielectric constant, and $f(\mathrm{~Hz})$ the operating frequency [12]. The other quantities appearing in (16)-(19) are given in Table 4.

In Figures 6 and 7, the red curves show results using Peplinski's principle. Blue curves show the results when both Peplinski (background medium) and multiple scattering are considered. The radius considered for the spheres is $a \approx$ $1.10 \mathrm{~cm}, c=0.2$ and $c=0.4$. Figure 6 shows the graphs of the phase velocity and the loss tangent versus the frequency when the permittivity of the background medium is given by (15) with $5 \%$ VWC. This background is introduced in (10) to yield the wave number that combines both Peplinski absorption (Maxwell-Garnett) and the multiple scattering. For a fixed frequency, it can be seen that the velocity decreases as the concentration increases and is much smaller than that in Figure 4. For the loss tangent, the blue and red curves decrease and show the same trend. Figure 7 shows a plot of the normalized phase velocity and loss tangent versus frequency with the volumetric water content (VWC) equal to $50 \%$. For the loss tangent, blue and red curves, although showing the same trend, dissociate as the frequency increases. The radius considered for the spheres is the same as that in Figure 6: $a \approx 1.10 \mathrm{~cm}, c=0.2$ and $c=0.4$. The comparisons of Figures 6 and 7 show that increasing the VWC lowers the phase velocity and the loss tangent, and this has a great influence on the wave propagation.

\section{Path Loss versus Distance}

Using the propagation constant in (12) derived from Peplinski's principle, the path loss $L_{p}^{P e}$ of an electromagnetic wave propagating in soil can be expressed as follows [28]:

$$
L_{p}^{P e}=6.4+20 \log (d)+20 \log (\Phi)+8.69 \varsigma d,
$$

where $d$ is the distance between sender and receiver and $8.69 \varsigma d$ is the additional attenuation caused by transmission. Our mixing of Peplinski's principle (12) with the multiple scattering theory given by (10) consists in replacing the 

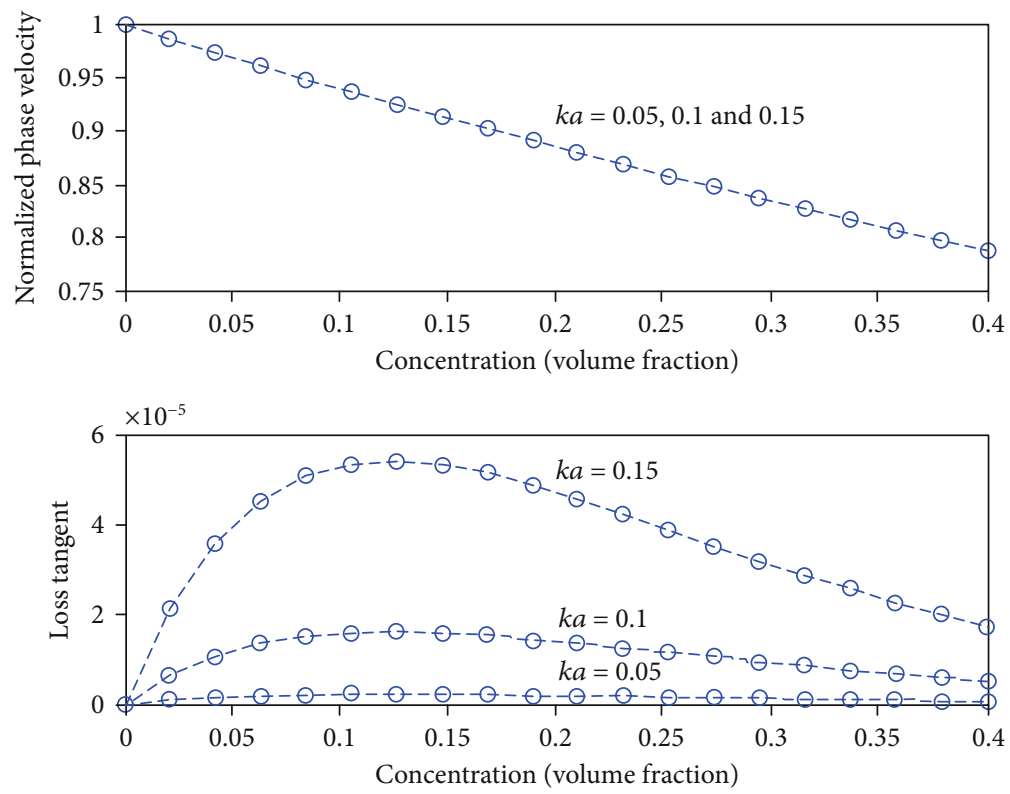

FIgURE 3: Normalized phase velocity and loss tangent versus concentration obtained from (10).
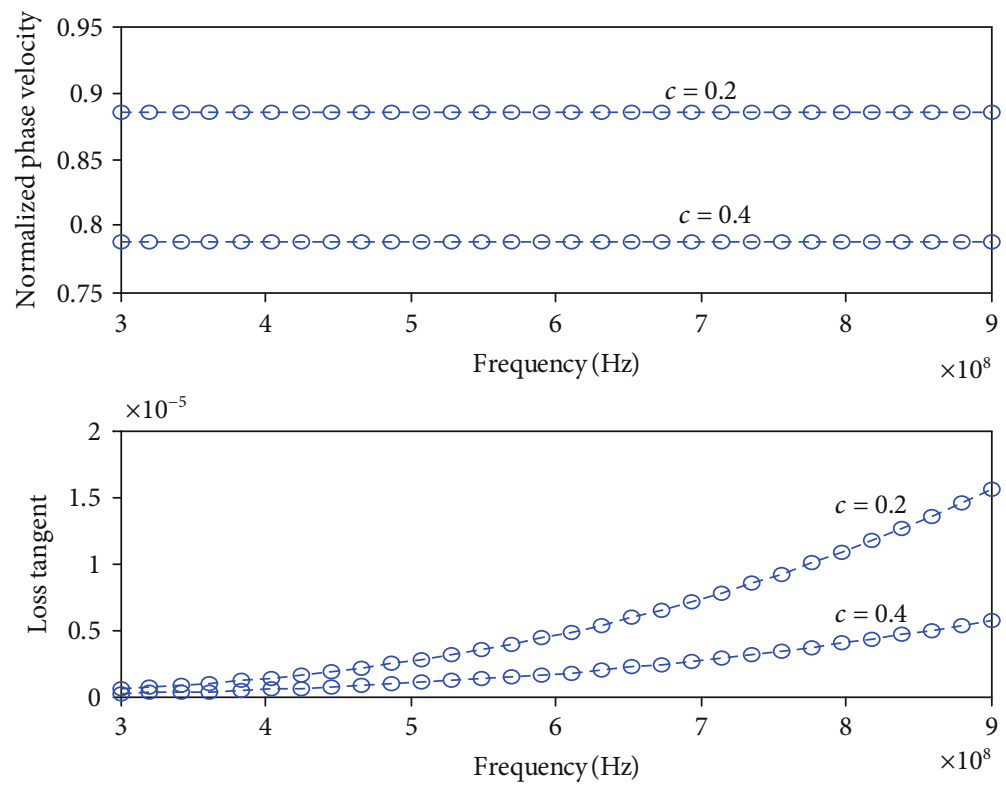

FIGURE 4: Normalized phase velocity and loss tangent versus frequency from (10) $a \approx 0.55 \mathrm{~cm}$.

background wave number $k=\omega \sqrt{\mu \varepsilon}$ in which $\varepsilon$ is the permittivity in free space by $k=\omega \sqrt{\mu\left(\varepsilon^{\prime}-i \varepsilon^{\prime \prime}\right)}$, where $\varepsilon^{\prime}$ and $\varepsilon^{\prime \prime}$ are given by (16) and (17). The resulting wave number is denoted by $K^{P e+m . s .}=k_{r}+i k_{i}$ (m.s. is used for multiple scattering). By making the correspondences $\omega \rightarrow k_{r}$ and $\varsigma \rightarrow-k_{i}$, the expression of the path loss in (20), which now includes multiple scattering, becomes

$$
L_{p}^{P e+\mathrm{m} . s .}=6.4+20 \log (d)+20 \log \left(k_{r}\right)-8.69 k_{i} d .
$$

The minus sign in (21) accounts for the fact that when propagating in the direction of increasing $x$, we have $e^{i \omega x} e^{\varsigma x}$ $e^{-i \omega t}$ using (12) and $e^{i k_{r} x} e^{-k_{i} x} e^{-i \omega t}$ using the wave number from our mixing formula.

In Figure 8, the red curves are obtained from Peplinski's principle and the blue curves from the combination of Peplinski and multiple scattering. The VWC in Figure 8(a) is $5 \%$, and in Figure $8(\mathrm{~b})$, it is $50 \%$. The radius considered for the spheres is once again $a \approx 1.10 \mathrm{~cm}, c=0.2$. The figure shows the path losses $L_{p}^{P e}$ and $L_{p}^{P e+m . s .}$ (in $\mathrm{dB}$ ) versus the distance $d$ between sender and receiver, at two typical IoT frequencies of $433 \mathrm{MHz}$ and $868 \mathrm{MHz}$. The data for the considered average soil types are as described in Table 4 . As 

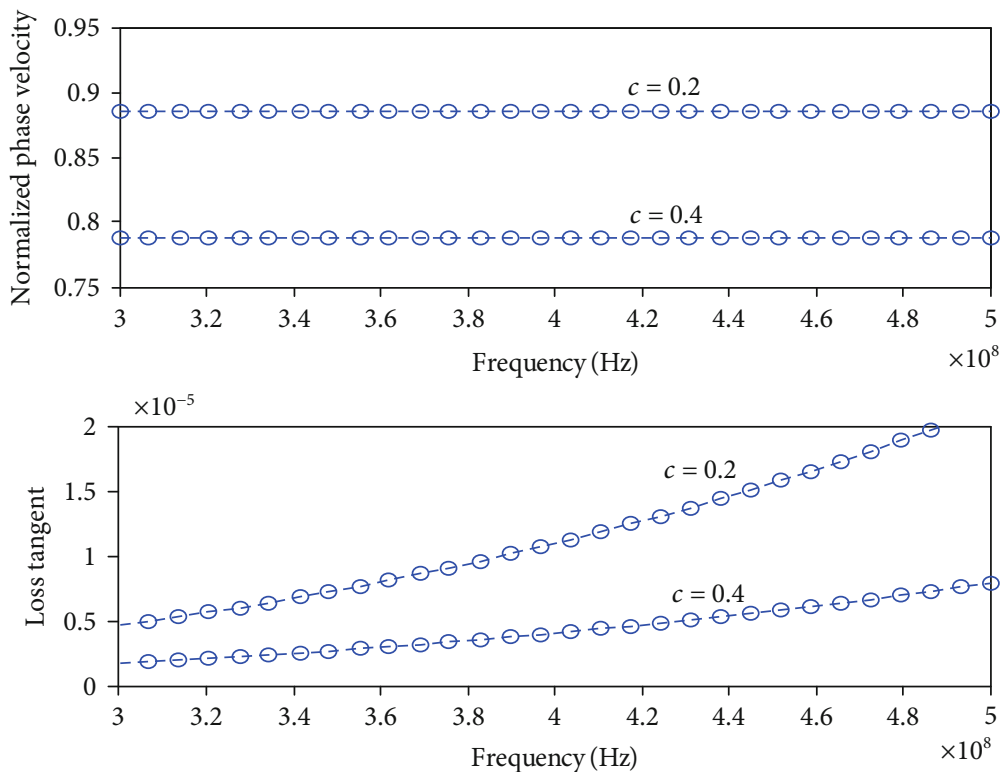

FIgURE 5: Normalized phase velocity and loss tangent versus frequency from (10) $a \approx 1.10 \mathrm{~cm}$.

TABLE 3: The physical parameters used for computing the wave number obtained by QCA.

\begin{tabular}{lcc}
\hline Symbol & Quantity & Value and units \\
\hline$c$ & Volume fraction & 0.2 or 0.4 \\
$\varepsilon_{p}$ & Relative permittivity in spheres & $3.2+i 0\left(\mathrm{~F} \mathrm{~m}^{-1}\right)$ \\
$\varepsilon$ & Background permittivity & $8.854 \times 10^{-12}\left(\mathrm{~F} \mathrm{~m} \mathrm{~m}^{-1}\right)$ \\
$a$ & Radius of spheres & $0.011(\mathrm{~m})$ (frequency up to 500 $\mathrm{MHz})$ \\
& & $0.055(\mathrm{~m})$ (frequency up to $900 \mathrm{MHz})$ \\
\hline
\end{tabular}

TABle 4: The physical parameters used for computing propagation constants from Peplinski's principle.

\begin{tabular}{|c|c|c|}
\hline Symbol & Quantity & Value and units \\
\hline$\rho_{b}$ & Bulk density of the soil & $1.5\left(\mathrm{~g} / \mathrm{cm}^{3}\right)$ \\
\hline$\rho_{s}$ & Bulk density of the solid soil particles & $2.66\left(\mathrm{~g} / \mathrm{cm}^{3}\right)$ \\
\hline$m_{v}$ & Volumetric water content (VWC) or moisture & $5 \%$ or $50 \%$ \\
\hline$\alpha^{\prime}$ & Soil-type empirically determined constant & 0.65 \\
\hline$\beta^{\prime}$ & Soil-type empirically determined constant with $C$ clay fraction and $S$ sand fraction & $1.2748-0.519 S-0.152 C$ \\
\hline$\beta^{\prime \prime}$ & Soil-type empirically determined constant & $1.3379-0.603 S-0.166 C$ \\
\hline$\varepsilon_{s}$ & Relative complex dielectric constant of the mixture of soil and water & $\left(1.01+0.44 \rho_{s}\right)^{2}-0.062$ \\
\hline$\tau_{w}$ & Relaxation time of water & $8 \times 10^{-12}$ \\
\hline$\varepsilon_{w 0}$ & Static dielectric constant of water & $80.4\left(\mathrm{~F} \mathrm{~m}^{-1}\right)$ \\
\hline$\varepsilon_{w \infty}$ & High-frequency limit of $\varepsilon_{f w}^{\prime}$ & $5.0\left(\mathrm{~F} \mathrm{~m}^{-1}\right)$ \\
\hline$\sigma_{\text {eff }}$ & Effective conductivity depending on soil texture & $0.046+0.220 \rho_{b}-0.411 S+0.661 C$ \\
\hline$\varepsilon_{0}$ & Permittivity constant of free space & $8.854 \times 10^{-12}\left(\mathrm{~F} \mathrm{~m}^{-1}\right)$ \\
\hline$\mu$ & Magnetic permeability & $4 \pi \times 10^{-7}\left(\mathrm{H} \mathrm{m}^{-1}\right)$ \\
\hline
\end{tabular}

expected, we observe that the path losses increase with increasing distance $d$. Furthermore, the increase in operating frequency $f$ leads to the increase in path loss. This analysis motivates the need to operate at the lowest possible frequencies in the soil medium in all cases, whether with the Peplinski principle or with the combination of Peplinski 

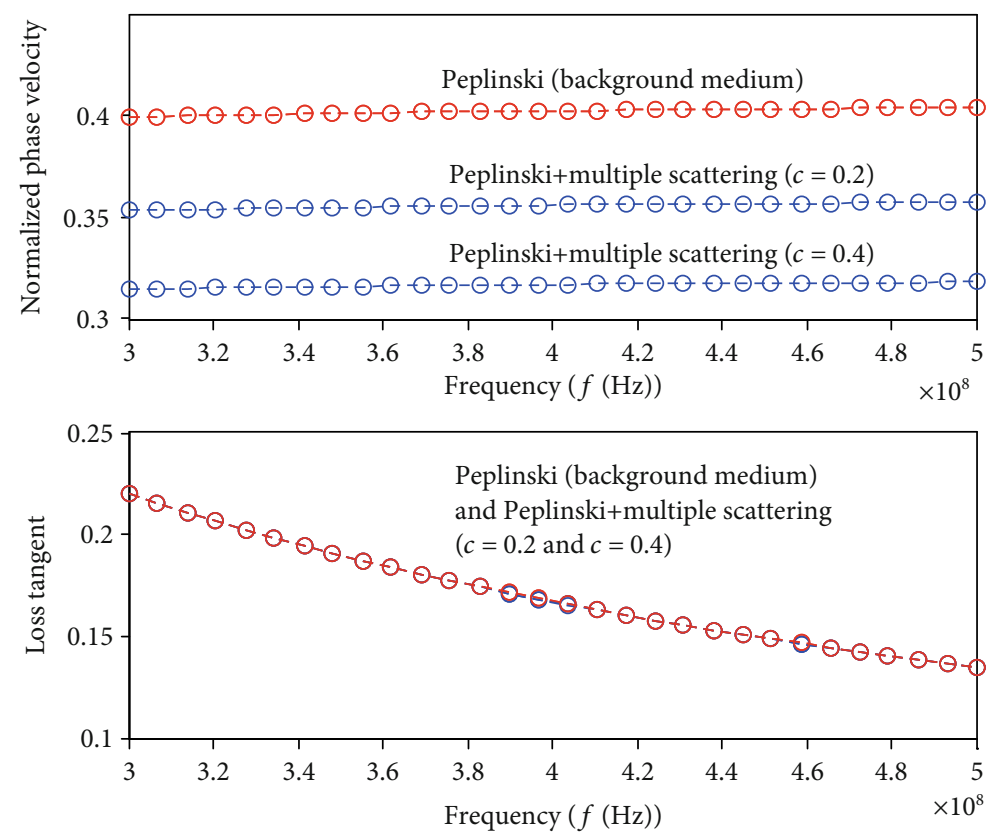

FIgURE 6: Normalized phase velocity and loss tangent versus frequency at VWC $=5 \%$.
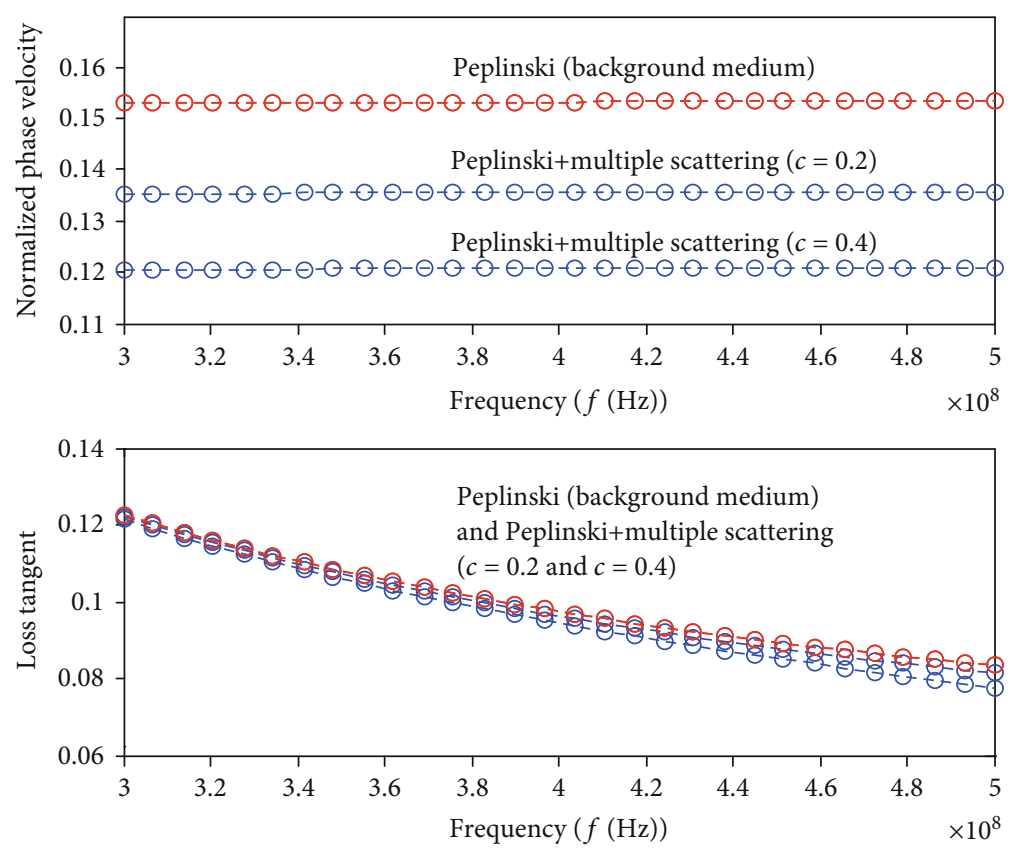

Figure 7: Normalized phase velocity and loss tangent versus frequency at VWC $=50 \%$.

and multiple scattering. Given the above results, a trade-off is required between operating at higher frequencies with a small antenna size but greater path loss and operating at a lower frequency using a bigger antenna but less path loss. An appropriate frequency range between 300 and $900 \mathrm{MHz}$ will be suitable for maintaining small antenna sizes [9]. This will ensure that the sensors remain concealed, a property which is distinctively useful for security applications.

We show the effect of VWC on the path loss for two values, $5 \%$ and $50 \%$. The path loss increases with higher pro- portions of VWC. This effect is particularly important since water content not only depends on the location of the network but also varies during different seasons and should therefore be considered in the design of WUSNs. The comparison between Figures 8(a) and 8(b) leads to the conclusion that it is not necessarily multiple scattering that provokes the high path loss values of $L_{p}^{P e+m . s .}$ as shown in Figure 8(a). For example, in Figure $8(\mathrm{~b})$, at the operating frequency of $868 \mathrm{MHz}, L_{p}^{P e+m . s .}<L_{p}^{P e}$. This shows that signal transmission is severely affected in soil containing not only random 


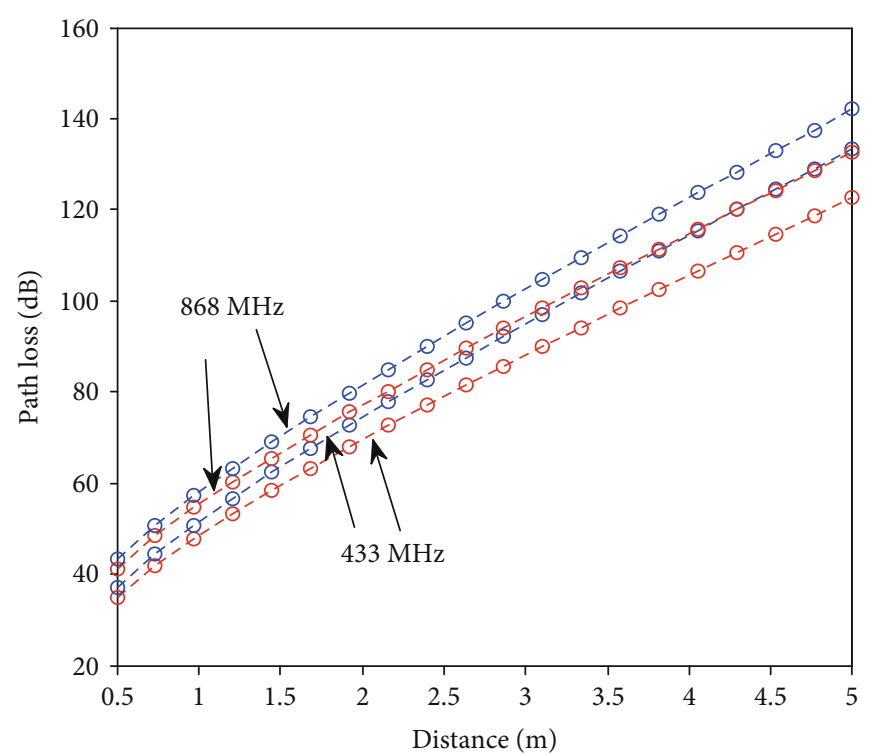

(a)

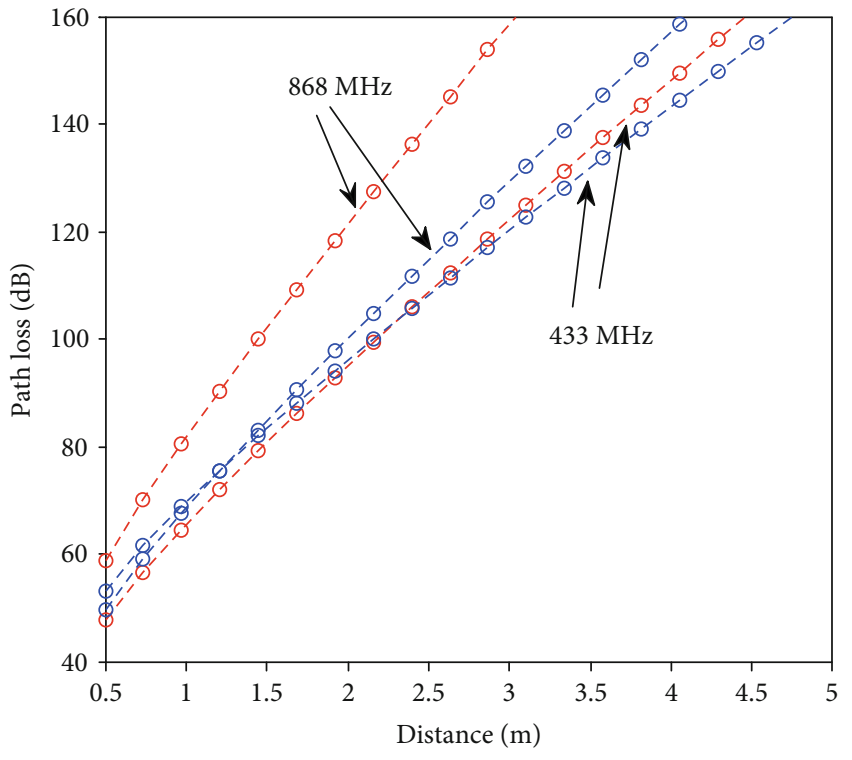

(b)

Figure 8: Path loss vs. distance and frequency (a) at VWC $=5 \%$ and (b) at VWC $=50 \%$.

distribution of spheres (stones) but also another important parameter which is water. We chose a relative permittivity $\varepsilon_{p}=3.2$ for the spheres. It must be noted that changing this value modifies the results discussed above. In particular, if $\varepsilon_{p}=1$, for example, $L_{p}^{P e}$ and $L_{p}^{P e+m . s}$. become identical as expected.

\section{Discussion}

In this paper, a new model of the effective wave number that accounts for absorption due to permittivity and multiple scattering occurring in soil because of the presence of buried obstacles such as stones, rocks, or pebbles has been proposed. From the analysis of normalized phase velocity and the loss tangent of wave propagation in soil as shown in Figures 4 and 5, it can be concluded that the wave velocity is less dependent on the concentration and the size of particles in soil. Furthermore, waves propagate faster in the soil medium with the lowest concentration of particles but with a higher loss tangent.

The graphs in Figures 6 and 7 indicate that a rise in the soil moisture causes a decrease of the phase velocity and the decay of the loss tangent, which diverge slightly as the frequency increases. In a nutshell, higher volumetric water content in soil reduces wave velocity leading to very slow wave propagation in soil.

Multiple scattering effects combined with Peplinski's principle enabled us to derive a mixed model of the effective wave number in soil, which accounts for both moisture and particle with a spherical shape. The numerical results show how the integration of the multiple scattering in the analysis modifies the path loss, which is a very important performance indicator for underground communication and adequate for the implementation of wireless underground sensor networks (WUSN).

\section{Conclusion}

In this paper, we have compared the wave number based upon a multiple scattering model in a dense medium soil with predictions based on the semiempirical model of Peplinski. The soil is assumed to contain a random distribution of scatterers or particles, which makes the estimation of the signal propagation in that medium quite challenging. By combining multiple scattering effect and Peplinski's principle, we derive a mixed model of the effective wave number in soil which accounts for both moisture and stones (of spherical shape). The numerical results show how the integration of the multiple scattering in the analysis modifies the path loss which is an important parameter for any wireless communication system and in the design and conceptualization of wireless underground sensor networks (WUSNs).

\section{Data Availability}

The data used to support the simulation results and the findings of this study are included within the article.

\section{Conflicts of Interest}

The authors declare that there is no conflict of interest regarding the publication of this paper.

\section{Acknowledgments}

The authors acknowledge the Department of Computer Science at the University of Ghana and the Department of Information Technology, UPSA, for their support. Special thanks are due to Laboratoire Ondes et Milieux complexes (LOMC) UMR CNRS 6294, University of Le Havre, France. 


\section{References}

[1] D. W. Sambo, A. Forster, and B. O. Yenke, "Wireless underground sensor networks path loss model for precision agriculture (WUSNPLM)," IEEE Sensors Journal, vol. 20, no. 10, pp. 5298-5313, 2020.

[2] A. Salam, "Subsurface MIMO: a beamforming design in internet of underground things for digital agriculture applications," Journal of Sensors and Actuator Networks, vol. 8, no. 3, p. 41, 2019.

[3] A. Villa-Henriksen, G. T. C. Edwards, L. A. Pesonen, O. Green, and C. A. G. Sørensen, "Internet of things in arable farming: implementation, applications, challenges and potential," Journal of Biosystems Engineering, vol. 191, pp. 60-84, 2020.

[4] H. Huang, J. Shi, F. Wang, D. Zhang, and D. Zhang, "Theoretical and experimental studies on the signal propagation in soil for wireless underground sensor networks," Sensors, vol. 20, no. 9, article 2580, 2020.

[5] U. Raza and A. Salam, "Wireless underground communications in sewer and stormwater overflow monitoring: radio waves through soil and asphalt medium," Information, vol. 11, no. 2, p. 98, 2020.

[6] M. C. Vuran and I. F. Akyildiz, "Channel model and analysis for wireless underground sensor networks in soil medium," Physical Communication, vol. 3, no. 4, pp. 245-254, 2010.

[7] K. Arshad, F. Katsriku, and A. Lasebae, "Radiowave VHF propagation modelling in forest using finite elements," in Proceedings -2006 International Conference on Information and Communication Technologies, pp. 2146-2149, Damascus, Syria, 2005.

[8] K. Arshad, F. Katsriku, and A. Lasebae, "Modeling obstructions in straight and curved rectangular tunnels by finite element approach," Journal of Electrical Engineering-Bratislava, vol. 59, no. 1, pp. 09-13, 2007.

[9] K. Arshad, F. Katsriku, and A. Lasebae, "Effects of different parameters on attenuation rates in circular and arch tunnels," Piers Online, vol. 3, no. 5, pp. 607-611, 2006.

[10] H. T. Friis, "A note on a simple transmission formula," Proceedings of the IRE, vol. 34, no. 5, pp. 254-256, 1946.

[11] Z. Sun and I. F. Akyildiz, "Magnetic induction communications for wireless underground sensor networks," IEEE Transactions on Antennas and Propagation, vol. 58, no. 7, pp. 24262435, 2010.

[12] X. Tan, Z. Sun, and I. F. Akyildiz, "Wireless underground sensor networks: MI-based communication systems for underground applications," IEEE Antennas and Propagation Magazine, vol. 57, no. 4, pp. 74-87, 2015.

[13] A. Salam, M. C. Vuran, and S. Irmak, "Pulses in the sand: impulse response analysis of wireless underground channel," in IEEE INFOCOM 2016-The 35th Annual IEEE International Conference on Computer Communications, San Francisco, CA, USA, 2016.

[14] F. H. Liedmann, C. Holewa, and C. Wietfeld, "The radio field as a sensor-a segmentation based soil moisture sensing approach," in 2018 IEEE Sensors Applications Symposium (SAS), Seoul, South Korea, 2018.

[15] N. R. Peplinski, F. T. Ulaby, and M. C. Dobson, "Dielectric properties of soils in the $0.3-1.3-\mathrm{GHz}$ range," IEEE Transactions on Geoscience and Remote Sensing, vol. 33, no. 3, pp. 803-807, 1995.
[16] L. K. Tsang, J. A. Kong, and K.-H. Ding, "Scattering and emission by layered media," in Scattering of Electromagnetic Waves: Theories and Applications, pp. 200-229, Wiley, New York, 1st edition, 2000.

[17] L. Tsang, J. A. Kong, K.-H. Ding, and C. O. Ao, "Particle positions for dense media characterizations and simulations," in Scattering of Electromagnetic Waves: Numerical Simulations, pp. 403-451, Wiley, New York, 1st edition, 2001.

[18] L. Tsang and J. A. Kong, "Quasi-crystalline approximation in dense medium scatteing," in Scattering of Electromagnetic Waves: Advanced Topics, pp. 245-319, Wiley, New York, 1st edition, 2001.

[19] M. C. Vuran, A. Salam, R. Wong, and S. Irmak, "Internet of underground things in precision agriculture: architecture and technology aspects," Ad Hoc Networks, vol. 81, no. 1, pp. 160-173, 2018.

[20] I. F. Akyildiz, "Wireless sensor networks in challenged environments such as underwater and underground," in Proceedings of the 17th ACM International Conference on Modeling, Analysis and Simulation of Wireless and Mobile Systems, Montreal, QC, Canada, 2014.

[21] M. A. Akkaş and R. Sokullu, "Wireless underground sensor networks: channel modeling and operation analysis in the terahertz band," International Journal of Antennas and Propagation, vol. 2015, 12 pages, 2015.

[22] I. F. Akyildiz, Z. Sun, and M. C. Vuran, "Signal propagation techniques for wireless underground communication networks," Physical Communication, vol. 2, no. 3, pp. 167-183, 2009.

[23] I. C. Dumitrache, S. I. Caramihai, I. S. Sacala, and M. A. Moisescu, "A cyber physical systems approach for agricultural enterprise and sustainable agriculture," in Proceedings -2017 21st International Conference on Control Systems and Computer (CSCS), Bucharest, Romania, 2017.

[24] M. Richardson Ansah, R. A. Sowah, J. Melià-Seguí, F. A. Katsriku, X. Vilajosana, and W. Owusu Banahene, "Characterising foliage influence on LoRaWAN pathloss in a tropical vegetative environment," IET Wireless Sensor Systems, vol. 10, no. 5, pp. 198-207, 2020.

[25] Y. S. Lei, P. Siqueira, and R. Treuhaft, "A dense medium electromagnetic scattering model for the InSAR correlation of snow," Radio Science, vol. 110, no. 1, pp. 461-480, 2016.

[26] L. Tsang, J. A. Kong, and R. T. Shin, Theory of Microwave Remote Sensing, New York, 1985.

[27] J. K. Percus and G. J. Yevick, "Analysis of classical statistical mechanics by means of collective coordinates," Physical Review, vol. 110, no. 1, pp. 1-13, 1958.

[28] L. Li, M. C. Vuran, and I. F. Akyildiz, "Characteristics of underground channel for wireless underground sensor networks," The 6th Annual Mediterranean Ad Hoc Networking Workshop, pp. 92-99, 2007. 